\title{
Avaliação da influência da relação mássica monômero (metacrilato de metila) / cera (palmitato de cetila) e da metodologia de processo na preparação de nanocápsulas contendo material de mudança de fase via polimerização em emulsão
}

\author{
Evaluation of the influence of the monomer \\ (methyl methacrylate) / wax (cetyl palmitate) \\ and process methodology on the preparation \\ of nanocapsules containing phase change \\ material via emulsion polymerization
}

\author{
Fernanda Barberato $^{1}$, Fernando Soares de Lima ${ }^{2}$, Kleber Lanigra Guimarães ${ }^{2}$ \\ Adriano Marim de Oliveira ${ }^{2}$
}

\footnotetext{
${ }^{1}$ Universidade Nove de Julho - Departamento de Ciências Exatas, CEP: 01156-050, São Paulo, São Paulo, Brasil.

${ }^{2}$ Instituto de Pesquisas Tecnológicas do Estado de São Paulo - Núcleo de Bionanomanufatura, CEP: 05508-901, São Paulo, São Paulo, Brasil.

e-mail: fe.barberato@hotmail.com
}

\begin{abstract}
RESUMO
Neste trabalho, foram produzidas nanocápsulas contendo material de mudança de fase ( "Phase Change Material” - PCM) por meio do processo de encapsulação via polimerização em emulsão, utilizando a cera palmitato de cetila como material encapsulado e o monômero metacrilato de metila como encapsulante, através de duas metodologias de processo distintas. A relação mássica cera/monômero também foi avaliada na produção das nanocápsulas. As amostras foram submetidas a caracterizações, com o objetivo de avaliar a estabilidade e integridade física das nanocápsulas após secagem em estufa, seu comportamento térmico (DSC), seus aspectos morfológicos (MEV), tamanho médio de partícula, conteúdo de sólidos e potencial operacional cíclico. Analisando os resultaos obtidos observou-se que tanto a metodologia de processo de produção quanto à variação da relação cera/monômero influenciaram diretamente na formação das nanocápsulas e no tamaho médio das partículas, sendo que as amostras produzidas através do Método II apresentaram melhores resultados quanto à formação das nanocápsulas com tamanho de partícula da ordem de $200 \mathrm{~nm}$, com elevada relação cera/monômero e estáveis a processos cíclicos de temperatura.
\end{abstract}

Palavras-chave: Polimerização em emulsão, nanocápsulas, PCM.

\begin{abstract}
In this work, nanocapsules containing Phase Change Material (PCM) were produced by means of the encapsulation process via emulsion polymerization, using the cetyl palmitate wax as encapsulated material and the methyl methacrylate monomer as encapsulant, through two different process methodologies. The wax / monomer mass ratio was also evaluated in the production of the nanocapsules. The samples were submitted to characterizations, with the objective of evaluating the stability and physical integrity of the nanocapsules after drying in the stove, their thermal behavior (DSC), their morphological aspects (SEM), average particle size, solids content and cyclic operational potential. Analyzing the obtained results, it was observed that both the production process methodology and the variation of the wax / monomer ratio directly influenced the formation of the nanocapsules and the average particle size, and the samples produced through Method II presented better results regarding formation of the nanocapsules with particle size of the order of $200 \mathrm{~nm}$, with high wax / monomer ratio and stable to cyclic temperature processes.
\end{abstract}

Keywords: Emulsion polymerization, nanocapsules, PCM. 


\section{INTRODUÇÃO}

$\mathrm{O}$ armazenamento de energia é um fator muito importante que tem como objetivo conservar e melhorar o aproveitamento da energia utilizada, uma vez que muitas fontes de energia são intermitentes na natureza[1-2]. $\mathrm{O}$ uso de materiais com alta capacidade de absorção e armazenamento de energia térmica, chamados de "Phase Change Materials" (PCM) ou materiais de mudança de fase, tem recebido grande atenção, já que os PCMs são uma forma de reaproveitamento da energia consumida, pois são materiais específicos, que se diferenciam dos materiais convencionais devido à sua maior capacidade de absorver e liberar calor a uma temperatura quase constante. São capazes de armazenar muito mais calor por unidade de volume do que os demais materiais que também sofrem processos de transformação de fase, como a água por exemplo [3-5].

$\mathrm{O}$ método de armazenamento de energia sob a forma de calor latente permite uma reserva com uma maior densidade energética, e em uma gama de temperatura mais estreita, quando comparado com o método de armazenamento de calor sensível [5-7]. Durante o processo de transformação de fase, inicialmente ocorre a absorção da energia fornecida ao material até o PCM atingir a temperatura em que acontece a mudança de fase, ou seja, atinge sua temperatura de fusão, ele então continua absorvendo grandes quantidades de calor, mesmo à temperatura constante, até que todo o material seja transformado para a fase líquida, sendo que esta energia envolvida no processo de transformação de fase é o calor latente de fusão. Quando ocorre o processo inverso, ou seja, a temperatura externa diminui e o calor absorvido pelo PCM é devolvido ao ambiente, o PCM se solidifica, liberando seu calor latente armazenado [6-7].

Considerando o tipo de transformação que o PCM sofre, temos três classificações, PCMs sólidosólido, PCMs sólido-líquido e PCMs líquido-gás. Dentre os três tipos o mais apropriado para o armazenamento de energia térmica é o PCM sólido-líquido. Dentre os PCMs sólido-líquido têm os PCMs inorgânicos (sais hidratados), os PCMs orgânicos (parafinas e não parafinas - ácidos graxos, ésteres, álcoois e glicóis-) e os PCMs eutéticos. Os PCMs possuem temperaturas de transição que se encontram no intervalo de temperatura de $-5^{\circ} \mathrm{C}$ até a temperatura de $190{ }^{\circ} \mathrm{C}$, porém grande parte das parafinas e não parafinas tem sua temperatura de transição dentro do intervalo de conforto humano, na faixa de temperatura de $20^{\circ} \mathrm{C}$ a $30^{\circ} \mathrm{C}$ [11-12]. É interessante que as temperaturas de fusão e solidificação sejam próximas para que com uma pequena variação da temperatura o PCM sofra os processos de transição [7-10]. A quantidade específica de calor que cada PCM é capaz de armazenar e posteriormente liberar varia de acordo com a temperatura de fusão, sendo que, quanto maior a temperatura de fusão, maior a quantidade de calor absorvido e consequentemente liberado.

Recentemente explorou-se a possibilidade de uso de tecnologias de encapsulação já consagradas para outros segmentos industriais (ex. fármacos e medicamentos, agroquímicos, etc.) para desenvolvimento de uma nova categoria / geração de PCMs encapsulados que apresentam como principal benefício proteger o PCM contra as influências do ambiente externo, aumentar a área de transferência de calor, a ação do revestimento como barreira, não permitindo a variação do volume do material do núcleo durante a mudança de fase, mantendo capacidade de operação cíclica do material [13-15]. Os PCMs encapsulados são revestidos por polímeros naturais ou sintéticos, onde o tamanho das cápsulas varia de menos de $1 \mu \mathrm{m}$ até $1000 \mu \mathrm{m}$, podendo ainda ultrapassar $1000 \mu \mathrm{m}$ [16-18].

Este trabalho visa apresentar um estudo que demonstre a viabilidade técnica de obtenção de PCMs nanoencapsulados pelo processo de polimerização em emulsão, destacando a importância da metodologia utilizada no processo de produção das nanocápsulas e a influência da variação da concentração de material ceroso e de monômero na formação dos PCMs encapsulados. As amostras produzidas foram avaliadas através das técnicas de difração de raios laser para análise do tamanho médio de partículas, termogravimetria auxiliada por luz halógena para análise do teor de sólidos, calorimetria exploratória diferencial (DSC) e microscopia eletrônica de varredura de alta resolução (MEV) a fim de evidenciar a formação dos PCMs encapsulados e confirmar seu potencial de uso como agente regulador de temperatura para operação em regimes cíclicos.

\section{MATERIAIS E MÉTODOS}

\subsection{Materiais}

Os materiais utilizados nas formulações como PCM foram a cera palmitato de cetila (CPC, Dhaymers) e o ácido esteárico (AE, Synth). Como monômero utilizou-se o metacrilato de metila (MMA, BASF S.A - grau técnico) e como monômeros funcionais foram testados o ácido acrílico (AA, Aldrich, 99\%), o estireno sulfonato de sódio $\left(\mathrm{StySO}_{3} \mathrm{Na}\right.$, Aldrich) e o metacrilato de hidroxietila (HEMA, Aldrich, 97\%). Sílica coloidal 
(SC, NALCO BRASIL, 30,56\% m/m em água) foi utilizada como colóide protetor e o persulfato de potássio (PK, VETEC, 99\%) como iniciador da reação de polimerização.

\subsection{Métodos}

\subsubsection{Métodos de produção das microcápsulas de PCM:}

MÉTODO I - Neste primeiro método, esquematizado na Figura 1, a cera foi adicionada ao monômero, a frio, onde permanecem sob agitação magnética até que a dispersão da cera no monômero fosse completa. O monômero funcional foi então adicionado à suspensão sob agitação magnética. Posteriormente, a dispersão de sílica coloidal em água (1), seguida da dispersão de monômero, monômero funcional e cera (2) e da solução de iniciador e água (3), foram adicionadas ao reator já aquecido, sob agitação constante.

MÉTODO II - Neste segundo método, esquematizado na Figura 2, a dispersão de sílica coloidal em água (1) e cera (2) foram adicionadas ao reator, onde permanecem até que se completasse a fusão da cera e se emulsionasse no meio. Em seguida, o monômero e o monômero funcional (3) e o iniciador em água (4) foram adicionadas ao reator sob agitação e fluxo de nitrogênio constantes.

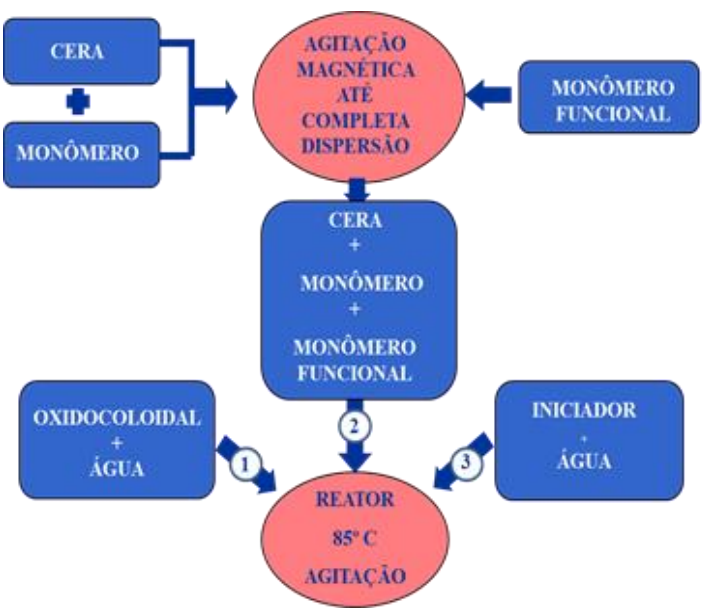

Figura 1: Fluxograma do Método I para a produção dos PCMs encapsulados.

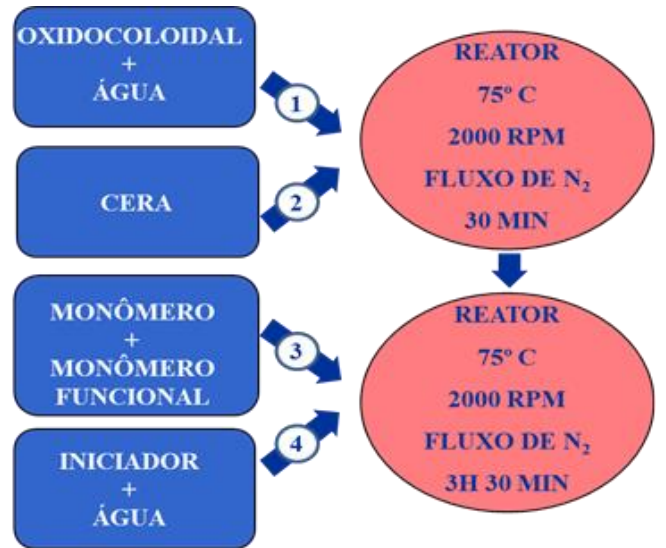

Figura 2: Fluxograma do Método II para a produção dos PCMs encapsulados.

A Tabela 1 a seguir apresenta as formulações empregadas nas reações de encapsulação dos PCMs com cada um dos métodos apresentados.

Tabela 1: Formulação das reações empregadas nas reações de encapsulação dos PCMs estudados.

\begin{tabular}{|c|c|c|c|c|c|c|c|c|c|c|c|}
\hline \multirow{3}{*}{$\begin{array}{l}\text { MÉTO- } \\
\text { DO }\end{array}$} & AMOSTRA & \multicolumn{9}{|c|}{ REAGENTES } & \multirow{2}{*}{$\begin{array}{c}\text { Monô- } \\
\text { mero } \\
/ \text { Cera } \\
(\mathrm{m} / \mathrm{m})\end{array}$} \\
\hline & PCM & $\begin{array}{c}\text { MMA } \\
(\mathrm{g})\end{array}$ & $\begin{array}{c}\text { CPC } \\
(g)\end{array}$ & $\begin{array}{l}A E \\
(g)\end{array}$ & $\begin{array}{l}\text { AA } \\
\text { (g) }\end{array}$ & $\begin{array}{l}\mathrm{StySO}_{3} \\
\mathrm{Na}(\mathrm{g})\end{array}$ & $\begin{array}{c}\text { HEMA } \\
\text { (g) }\end{array}$ & $\begin{array}{l}\text { SC } \\
\text { (g) }\end{array}$ & $\begin{array}{l}\text { PK } \\
\text { (g) }\end{array}$ & $\begin{array}{c}\mathrm{H} 2 \mathrm{O} \\
(\mathrm{g})\end{array}$ & \\
\hline & BRANCO & 18,00 & ----- & ----- & ----- & 0,21 & ----- & 2,51 & 0,43 & 92,99 & 18,00 \\
\hline \multirow{2}{*}{$\begin{array}{c}\text { Método } \\
\text { I }\end{array}$} & $\mathrm{I}-1$ & 18,09 & ----- & 2,02 & 0,44 & ----- & ----- & 2,53 & 0,41 & 95,15 & 8,95 \\
\hline & $I-2$ & 18,00 & ----- & 2,00 & ----- & 0,21 & ----- & 2,51 & 0,42 & 92,89 & 9,00 \\
\hline \multirow{7}{*}{$\begin{array}{l}\text { Método } \\
\text { II }\end{array}$} & II -1 & 13,11 & ----- & 7,03 & ---- & 0,40 & ----- & 5,03 & 0,81 & 176,08 & 1,86 \\
\hline & II -2 & 13,03 & 3,51 & ----- & ----- & 0,40 & ----- & 5,01 & 0,80 & 176,24 & 3,71 \\
\hline & $\mathrm{II}-3$ & 13,14 & 7,00 & ----- & ----- & 0,41 & ----- & 5,08 & 0,84 & 176,64 & 1,88 \\
\hline & $\mathrm{II}-4$ & 13,00 & 7,02 & ----- & 0,40 & ----- & ----- & 5,04 & 1,01 & 175,18 & 1,85 \\
\hline & II -5 & 13,01 & 7,10 & ---- & ---- & ----- & 0,40 & 5,01 & 0,83 & 178,35 & 1,83 \\
\hline & II -6 & 8,03 & 12,0 & ----- & ----- & 0,40 & ----- & 5,08 & 0,81 & 176,77 & 0,66 \\
\hline & II -7 & 4,04 & 16,0 & ----- & ----- & 0,40 & ----- & 5,01 & 0,83 & 178,50 & 0,25 \\
\hline
\end{tabular}


Utilizando a técnica de polimerização em emulsão, dois métodos diferentes foram estudados, sendo que a principal diferença entre eles se encontra na mudança da ordem de adição dos materiais no processo de reação. Em ambos os métodos foram utilizados os mesmos aparatos, sendo um reator encamisado de $350 \mathrm{~mL}$, com banho de aquecimento, um condensador acoplado ao reator, encamisado com banho de resfriamento, um agitador mecânico com velocidade de $400 \pm 10 \mathrm{rpm}$, cujo impelidor utilizado foi do tipo disco com lâminas planas (CAWLESS), e no caso do Método II, foi utilizado um tubo para injeção de nitrogênio, para manter a atmosfera inerte.

\subsubsection{Caracterização dos PCMs:}

Os PCMs foram caracterizados pela técnica de DSC, iniciando com uma rampa de aquecimento partindo da temperatura de $25^{\circ} \mathrm{C}$ até $100^{\circ} \mathrm{C}$ e posterior resfriamento da temperatura de $100^{\circ} \mathrm{C}$ à temperatura de $25^{\circ} \mathrm{C} \mathrm{com}$ uma taxa de aquecimento/resfriamento de $5^{\circ} \mathrm{C}$ por minuto, com fluxo de nitrogênio constante de $50 \mathrm{~mL} / \mathrm{min}$, utilizando cadinhos de alumina abertos, com uma massa de amostra entre 5-10 mg. A convenção da representação gráfica adotada pelo software $\operatorname{Star}^{\mathrm{e}}$ (Mettler Toledo) define que deslocamentos para cima da linha base são representativos de eventos exotérmicos (ex. recristalização), ao passo que deslocamentos para baixo são representativos de eventos endotérmicos (ex. fusão).

\subsubsection{Caracterizações dos PCMs encapsulados:}

Após a o processo de formação das nanocápsulas as amostras foram submetidas a secagem em estufa à temperatura de $90^{\circ} \mathrm{C}$ por um intervalo de tempo de cerca de 30 minutos ou até que o material apresentasse características coagulada ou de pó. As amostras foram depositadas sobre placas de petri de vidro e a secagem foi feita utilizando-se uma estufa de circulação de ar marca Nova Ética e modelo 400/3ND-300.

A caracterização das amostras quanto ao tamanho médio de partículas foi realizada utilizando-se equipamento da marca Beckman Coulter, modelo LS 230, onde a leitura foi realizada nas amostras dispersas em água. Para caracterizar as amostras quanto ao teor de sólidos, utilizou-se uma termobalança de luz halógena da marca Mettler Toledo, modelo HB43-S. Foram utilizadas massas de dispersão dos PCMs encapsulados entre 0,5 a $0,6 \mathrm{~g}$, aquecidas à temperatura de $110^{\circ} \mathrm{C}$, até que a massa residual da amostra sofresse uma variação menor que $0,1 \mathrm{~g}$ em seu resultado.

Os PCMs encapsulados foram também submetidos à análise térmica de DSC, antes e após a secagem em estufa, cujas análises foram conduzidas de acordo com a mesma programação de ensaio apresentada para a caracterização dos materiais utilizados como PCMs na sua forma pura. O equipamento utilizado foi o modelo DSC $822^{\mathrm{e}}$ da Mettler Toledo.

Para a caracterização da morfologia das amostras por microscopia eletrônica de varredura (MEV) adotou-se uma preparação, onde, primeiramente as amostras foram secas em estufa a $80^{\circ} \mathrm{C}$, o pó obtido foi depositado sobre uma fita dupla-face de cobre e recoberto por uma fina película de ouro. As análises foram realizadas em alto vácuo, com uma tensão de aceleração do feixe de elétrons de $20 \mathrm{kV}$. Foram feitas análises com ampliações de 25.000, 65.000, 80.000 e até 100.000 vezes de aumento.

\section{RESULTADOS E DISCUSSÃO}

Inicialmente foi produzida uma amostra referência (branco), com a ausência de material ceroso, a fim de avaliar o comportamento do sistema na presença somente do monômero, emulsificante e meio aquoso de dispersão. Os primeiros ensaios foram conduzidos empregando-se o Método I, utilizando o ácido esteárico como PCM. O ácido esteárico apresenta um pico de fusão próximo à temperatura de $60^{\circ} \mathrm{C}$, pico de recristalização em torno da temperatura de $50^{\circ} \mathrm{C}$ e capacidade de absorção de energia ( $\Delta \mathrm{H}$ fusão) da ordem de $-138,29 \mathrm{~J} / \mathrm{g}$ (Figura 3). Nestes experimentos a razão mássica entre o monômero e o ácido esteárico foi da ordem de nove vezes mais monômero.

Após a produção das primeiras amostras de PCM encapsulados via Método I, verificou-se que as amostras não secavam quando expostas à temperatura de $90{ }^{\circ} \mathrm{C}$ em estufa e ficavam com aspecto oleoso nesta temperatura, demonstrando que o PCM em questão não havia sido encapsulado e a cera estava sofrendo o processo de fusão livremente. A análise do teor de sólidos destas amostras demonstrou que o processo de polimerização ocorreu, ou seja, o monômero, que é volátil, foi convertido em polímero sólido, porém o aspecto oleoso das amostras secas em estufa indica que a cera não foi encapsulada pelo polímero.

Diante dos primeiros resultados obtidos das amostras produzidas pelo Método I foi avaliado um segundo método, denominado Método II, onde inicialmente manteve-se o ácido esteárico como PCM e variouse a proporção em massa de monômero em relação à cera, adotando-se a proporção de aproximadamente 1,85 vezes mais monômero a fim de verificar se a razão mássica entre os componentes poderia interferir no pro- 
cesso de encapsulação da cera. A primeira amostra produzida pelo Método II, denominada II-1, foi seca em estufa à temperatura de $90^{\circ} \mathrm{C}$, onde apresentou aspecto seco e formação de um pó, indicando que o material ceroso não estava livre, possivelmente foi encapsulado pelo polímero formado, pois nesta temperatura de $90^{\circ} \mathrm{C}$ o ácido esteárico estaria liquido caso não estivesse encapsulado.

Entre os dois métodos experimentais praticados, optou-se em dar prosseguimento aos testes utilizando o Método II. Optou-se ainda por alterar o ácido esteárico e avaliar outra cera como PCM, a palmitato de cetila, a qual apresenta pico de fusão bem definido à temperatura de $54^{\circ} \mathrm{C}$, pico de recristalização à temperatura de $43^{\circ} \mathrm{C}$ e entalpia de fusão ( $\Delta \mathrm{H}$ fusão) da ordem de $-172,79 \mathrm{~J} / \mathrm{g}$ (Figura 4), tratando-se, portanto, de um material com uma temperatura de fusão mais baixa e uma capacidade de absorção de energia no seu processo de fusão maior, quando comparada ao ácido esteárico.

Na produção dos PCMs encapsulados utilizando a cera palmitato de cetila segundo o Método II variou-se novamente as proporções em massa de monômero em relação à cera, aumentando a quantidade de cera na formulação, a fim de avaliar se o método permitiria a obtenção do PCM encapsulado mesmo com grandes quantidades de cera presente no sistema, e consequentemente aumentar o potencial de absorção de calor do produto final.

Com exceção da secagem em estufa, as demais caracterizações das amostras produzidas foram realizadas após lavagem com solvente orgânico, metanol, que não reage com o polímero, somente com a cera, a fim de retirar qualquer material ceroso residual externo às nanocápsulas. Após a lavagem as amostras foram novamente secas em estufa.

As Figuras 3 e 4 apresentam os perfis térmicos obtidos pela técnica de DSC para as amostras de ácido esteárico e da cera palmitato de cetila antes do processo de encapsulação, respectivamente, e a Tabela 2 apresenta os resultados obtidos em cada experimento de encapsulação dos PCMs realizado neste trabalho.

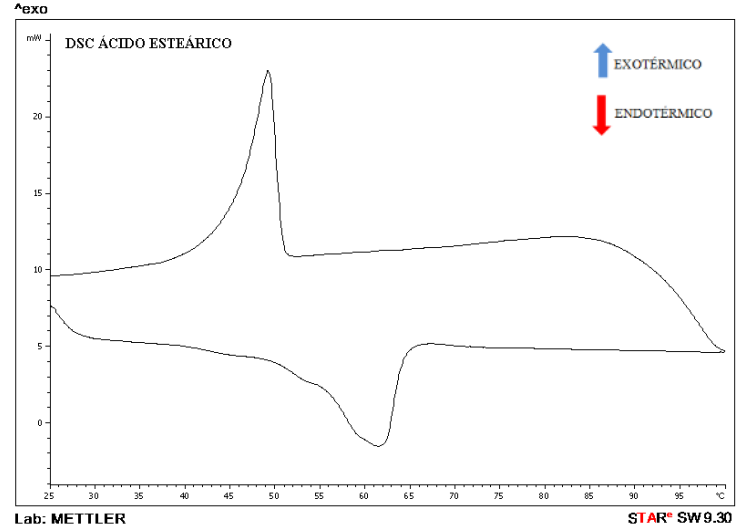

Figura 3: Perfil térmico de DSC do ácido esteárico.

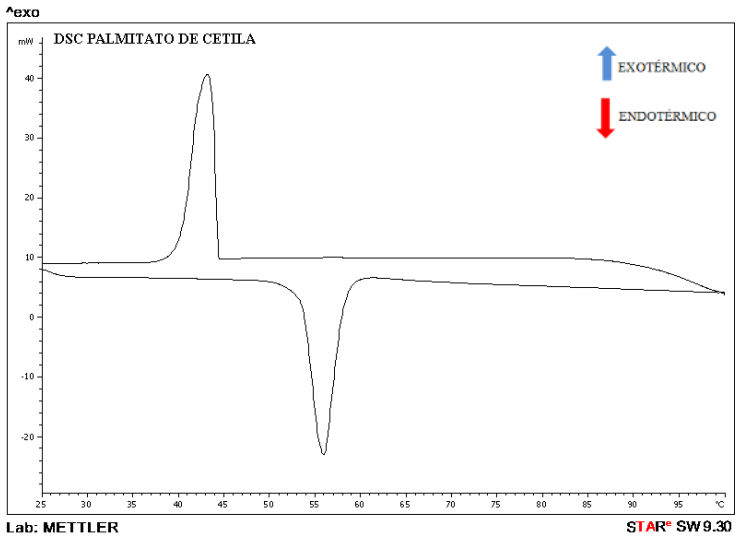

Figura 4: Perfil térmico de DSC da cera palmitato de cetila.

Tabela 2: Caracterizações dos PCMs encapsulados

\begin{tabular}{|c|c|c|c|c|c|c|c|c|c|}
\hline \multirow{3}{*}{ MÉTODO } & \multirow{2}{*}{$\begin{array}{c}\text { AMOSTRA } \\
\text { PCM }\end{array}$} & \multicolumn{2}{|c|}{ CARACTERÍSTICAS } & \multicolumn{6}{|c|}{ CARACTERIZAÇÕES } \\
\hline & & $\begin{array}{c}\text { Cera } \\
\text { presente }\end{array}$ & $\begin{array}{c}\text { Monômero/ } \\
\text { Cera } \\
(\mathbf{m} / \mathbf{m})\end{array}$ & $\begin{array}{c}\text { Secagem } \\
\text { na estufa } \\
\left(90^{\circ} \mathrm{C}\right)\end{array}$ & $\begin{array}{l}\text { TMP } \\
(\mathrm{nm})\end{array}$ & $\begin{array}{l}\text { TST } \\
(\%)\end{array}$ & $\begin{array}{l}\text { TSE } \\
(\%)\end{array}$ & $\begin{array}{c}\Delta H \text { fusão } \\
(\mathbf{J} / \mathbf{g})\end{array}$ & $\begin{array}{c}\text { Ponto } \\
\text { de } \\
\text { Fusão } \\
\text { (oC) }\end{array}$ \\
\hline & BRANCO & ----- & 18,00 & Pó & 164 & 17,02 & 14,59 & NA & NA \\
\hline \multirow{2}{*}{ Método I } & $\mathrm{I}-1$ & $\mathrm{AE}$ & 8,95 & Coagulado & 236 & 19,80 & 15,67 & NA & NA \\
\hline & $\mathrm{I}-2$ & $\mathrm{AE}$ & 9,00 & Coagulado & 126 & 18,45 & 18,36 & NA & NA \\
\hline \multirow{7}{*}{ Método II } & II -1 & $\mathrm{AE}$ & 1,86 & Pó & 3.970 & 11,06 & 8,54 & $-41,77$ & 54,54 \\
\hline & II -2 & $\mathrm{CPC}$ & 3,71 & Pó & 6.549 & 11,26 & 9,10 & $-23,45$ & 49,54 \\
\hline & II -3 & $\mathrm{CPC}$ & 1,88 & Pó & 20.240 & 11,30 & 9,35 & $-34,08$ & 53,95 \\
\hline & II -4 & $\mathrm{CPC}$ & 1,85 & Pó & 28.600 & 11,33 & 8,68 & $-46,52$ & 52,75 \\
\hline & II -5 & $\mathrm{CPC}$ & 1,83 & Pó & 16.700 & 11,17 & 9,28 & $-48,82$ & 53,13 \\
\hline & II -6 & $\mathrm{CPC}$ & 0,66 & Pó & 15.600 & 10,98 & 9,80 & $-82,18$ & 55,01 \\
\hline & II -7 & $\mathrm{CPC}$ & 0,25 & Pó & 12.890 & 11,14 & 10,30 & $-95,77$ & 57,32 \\
\hline
\end{tabular}


AE: Ácido esteárico; CPC: Cera Palmitato de Cetila; TMP: Tamanho Médio de Partículas; TST: Teor de Sólidos Teórico; TSE: Teor de Sólidos Experimental; EE: Eficiência de Encapsulação; NA: Não apresenta;

O aspecto das amostras após secagem na estufa foi o primeiro indicativo do processo de encapsulação dos PCMs, sendo que as amostras que se apresentaram na forma de pó, quando submetidas à caracterização por MEV, observou-se a formação das cápsulas e baseando-se na escala da micrografia, fica evidenciado que o tamanho das partículas é menor do que $1 \mu \mathrm{m}$, conforme apresentado nas Figuras 7 e 8.

Os resultados de tamanho médio de partículas obtidos por difração de raios laser apresentados na Tabela 2 apresentaram elevado grau de variação contemplando grandezas na ordem de nanômetros a micrômetros. As amostras produzidas pelo Método I apresentaram tamanhos menores, da ordem de nanômetros, provavelmente devido à ineficiência do processo de encapsulação, onde possivelmente ocorreu apenas a polimerização do monômero, sem formação de encapsulação. No caso das amostras produzidas segundo o Método II houve significativo aumento do tamanho médio de partículas, da ordem de micrômetros, possivelmente devido ao fato da maior quantidade de cera incorporada na formulação dos PCMs encapsulados.

Os valores de temperatura e entalpia de fusão apresentados na Tabela 2 foram experimentalmente determinados através dos perfis térmicos obtidos das análises de DSC das amostras. Os valores de $\Delta H$ de fusão apresentados na Tabela 2 indicam a presença do material ceroso nas amostras analisadas, sendo que quanto maior a relação cera/monômero, maior o $\Delta \mathrm{H}$ fusão determinado, exceto nas amostras produzidas pelo Método I que, por não apresentarem pico de fusão, não apresentam $\Delta \mathrm{H}$ de fusão, como também ocorre no caso da amostra de PCM-BRANCO, que por não conter material ceroso no sistema, não apresenta pico de fusão e nem $\Delta \mathrm{H}$ de fusão. As Figuras 5 e 6, respectivamente, representam as curvas de DSC da amostra PCM-I-2, que representa um perfil típico obtido para as amostras produzidas pelo Método-I, onde não se observa picos definidos de fusão e de recristalização, o que comprova que não há presença de material ceroso nessas amostras, e da amostra PCM-II-3, que representa um perfil térmico típico das amostras produzidas segundo o Método-II. Após as análises a amostra residual retida nos cadinhos não apresentou aspecto de material ceroso fundido.

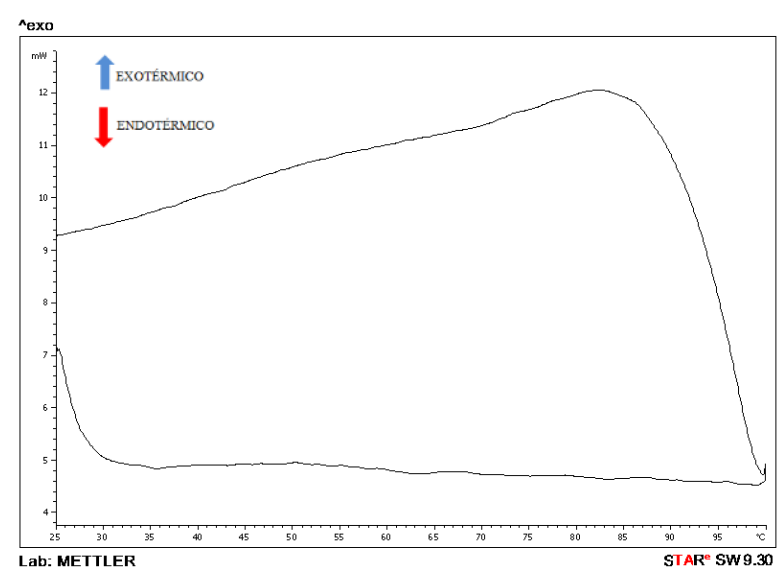

Figura 5: Perfil térmico de DSC da amostra PCM-I-2.

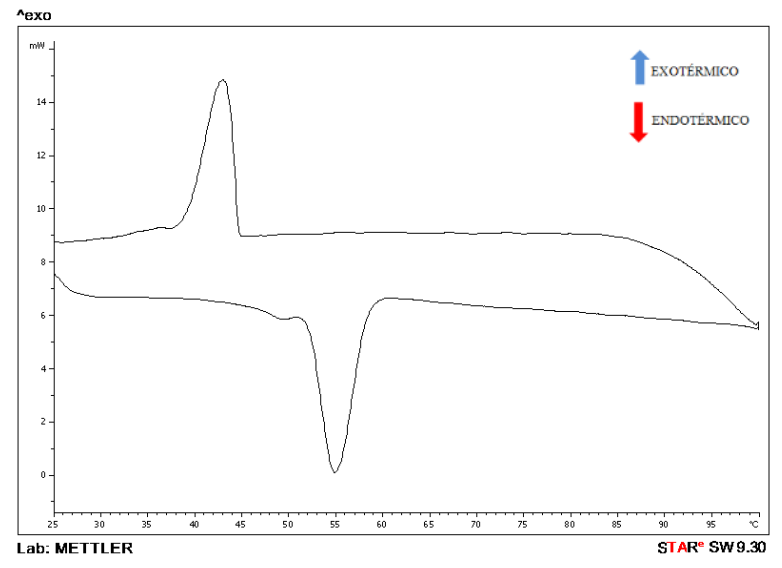

Figura 6: Perfil térmico de DSC da amostra PCM-II-3.

As imagens obtidas pela microscopia eletrônica de varredura (MEV) das amostras produzidas conforme o Método-II podem ser observadas nas Figuras 7 e 8, onde é possível confirmar a formação das cápsulas de PCM. Através da escala das imagens é possível observar que o tamanho real das cápsulas é da ordem de aproximadamente 200 nanômetros, o que nos permite concluir que o tamanho médio de partículas determinado pela técnica de difração a laser, cujos valores estão apresentados na Tabela 2, tratam-se das medidas dos aglomerados de partículas em escala nanométrica.

As imagens de MEV das amostras após serem submetidas ao ensaio de DSC, Figuras 8 e 10, comprovam a integridade das cápsulas mesmo após ciclos térmicos de aquecimento e resfriamento. Sendo assim, podemos também considerar que o perfil térmico apresentado nas análises térmicas de DSC refere-se ao material ceroso presente no interior das nanocápsulas. 

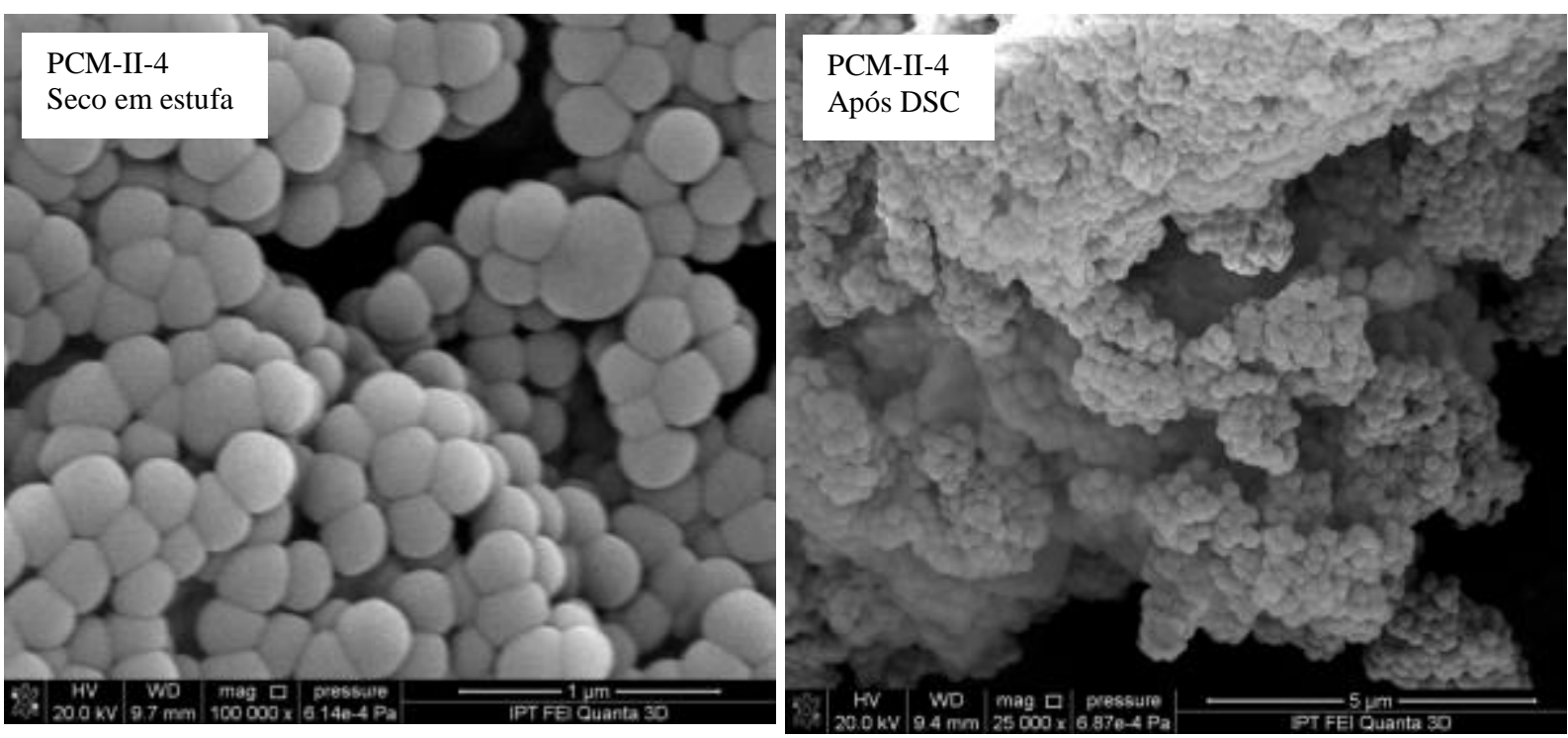

Figura 7: Micrografia com ampliação de 100000 vezes da amostra PCM-II-4 após secagem em estufa.

Figura 8: Micrografia com ampliação de 25000 vezes da amostra PCM-II-4 após análise de DSC.

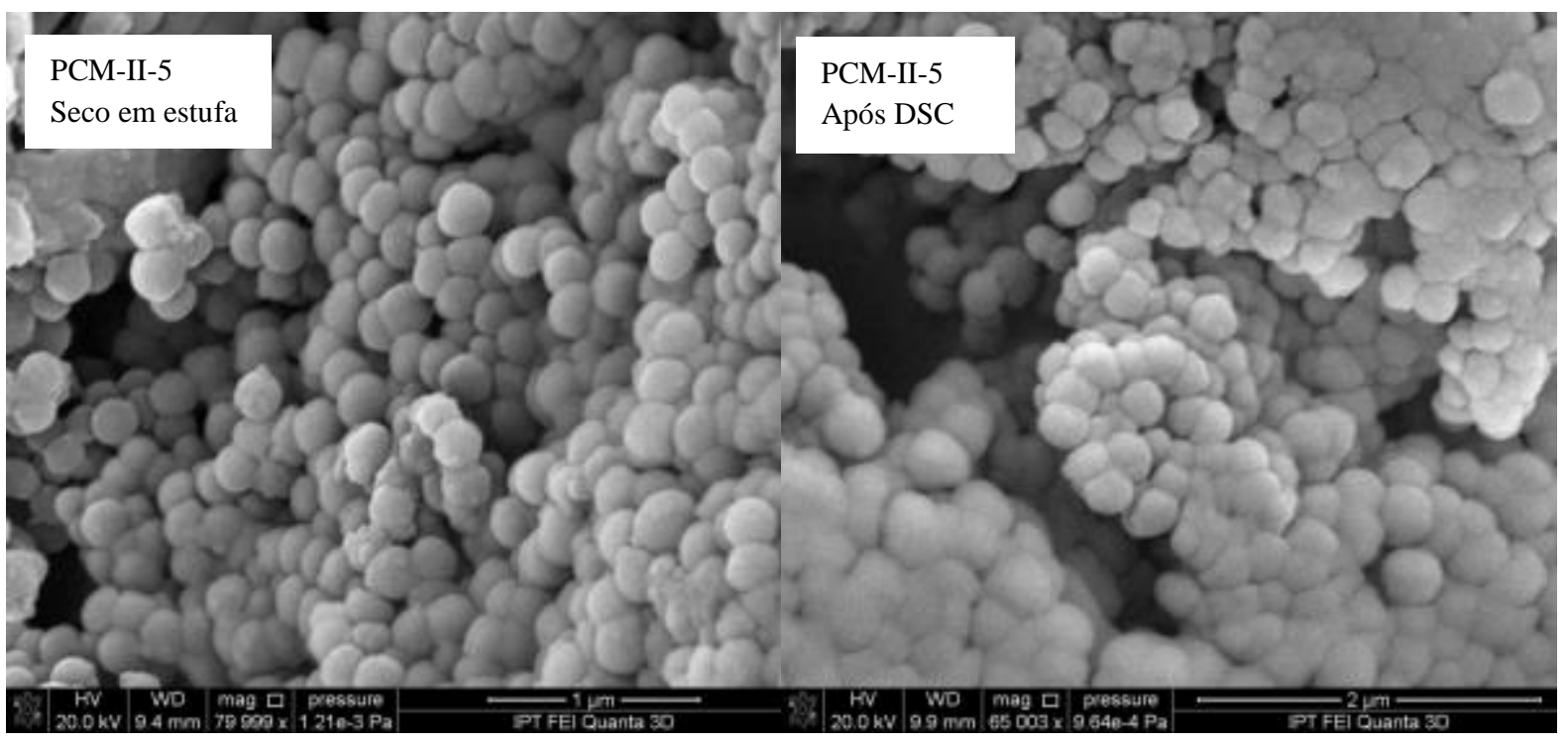

Figura 9: Micrografia com ampliação de 80000 vezes da

Figura 10: Micrografia com ampliação de 65000 vezes da amostra PCM-II-5 após secagem em estufa. amostra PCM-II-5 após análise de DSC.

A análise de teor de sólidos realizada na suspensão de nanocápsulas de PCM pode ser utilizada para determinar a eficiência da polimerização através da comparação do teor de sólidos teórico da reação com o experimental obtido, levando-se em consideração que a diferença entre o teor de sólidos teórico e experimental trata da volatilização do monômero e a não participação deste na reação. Para determinação da eficiência da polimerização foi utilizada a Equação1. A Tabela 3 apresenta os resultados obtidos de eficiência de polimerização do PCM encapsulado.

$E P=\frac{T S e-T S s m}{T S m} \times 100$

Onde:

EP - Eficiência de polimerização

TSe - Teor de sólidos experimental

TSsm - Teor de sólidos sem monômero

TSm - Teor de sólidos do monômero 
Tabela 3: Caracterização dos PCMs encapsulados quanto ao teor de sólidos e a eficiência de polimerização.

\begin{tabular}{|c|c|c|c|c|c|c|c|c|}
\hline \multirow[b]{2}{*}{$\begin{array}{l}\text { MÉTO- } \\
\text { DO }\end{array}$} & \multirow{2}{*}{$\begin{array}{c}\begin{array}{c}\text { AMOS } \\
\text { TRA }\end{array} \\
\\
\text { PCM }\end{array}$} & \multicolumn{2}{|c|}{ CARACTERÍSTICAS } & \multicolumn{5}{|c|}{ CARACTERIZAÇÃO } \\
\hline & & $\begin{array}{c}\text { Cera } \\
\text { presente }\end{array}$ & $\begin{array}{c}\text { Monôme- } \\
\text { ro/Cera } \\
(\mathrm{m} / \mathrm{m})\end{array}$ & $\begin{array}{c}\text { Teor de só- } \\
\text { lidos teórico } \\
\text { (\%) }\end{array}$ & $\begin{array}{l}\text { Teor de sóli- } \\
\text { dos experi- } \\
\text { mental } \\
(\%)\end{array}$ & $\begin{array}{l}\text { Teor de sóli- } \\
\text { dos do mo- } \\
\text { nômero (\%) }\end{array}$ & $\begin{array}{l}\text { Teor de só- } \\
\text { lidos sem } \\
\text { monômero } \\
(\%)\end{array}$ & $\begin{array}{c}\text { Eficiência } \\
\text { da poli- } \\
\text { meriza- } \\
\text { ção } \\
\text { (\%) }\end{array}$ \\
\hline \multirow{2}{*}{$\begin{array}{c}\text { Método } \\
\text { I }\end{array}$} & $\mathrm{I}-1$ & $\mathrm{AE}$ & 8,95 & 19,80 & 15,67 & 15,25 & 3,07 & 82,63 \\
\hline & $\mathrm{I}-2$ & $\mathrm{AE}$ & 9,00 & 18,45 & 18,36 & 15,69 & 2,75 & 99,47 \\
\hline \multirow{7}{*}{$\begin{array}{l}\text { Método } \\
\text { II }\end{array}$} & II -1 & $\mathrm{AE}$ & 1,86 & 11,06 & 8,54 & 6,76 & 4,31 & 62,65 \\
\hline & II -2 & $\mathrm{CPC}$ & 3,71 & 11,26 & 9,10 & 6,83 & 2,59 & 95,30 \\
\hline & $\mathrm{II}-3$ & $\mathrm{CPC}$ & 1,88 & 11,30 & 9,35 & 6,76 & 4,29 & 74,83 \\
\hline & II -4 & $\mathrm{CPC}$ & 1,85 & 11,33 & 8,68 & 6,53 & 4,62 & 62,17 \\
\hline & $\mathrm{II}-5$ & $\mathrm{CPC}$ & 1,83 & 11,17 & 9,28 & 6,63 & 4,30 & 75,04 \\
\hline & II -6 & $\mathrm{CPC}$ & 0,66 & 10,98 & 9,80 & 4,20 & 6,78 & 71,89 \\
\hline & $\mathrm{II}-7$ & $\mathrm{CPC}$ & 0,25 & 11,14 & 10,30 & 2,19 & 8,71 & 72,43 \\
\hline
\end{tabular}

Avaliando os resultados de eficiência de polimerização calculados, é possível correlacionar com os demais resultados apresentados anteriormente, onde se observa que, mesmo para as amostras produzidas com uma relação monômero/cera muito baixa, ou seja, grandes quantidades de cera presente no sistema, como é o caso das amostras PCM-II-3 a PCM-II-7, a porcentagem de eficiência de polimerização é alta, da ordem de $70 \%$, ocorrendo a formação das nanocápsulas, comprovada pelas demais técnicas de caracterização anteriormente apresentadas. Já as amostras PCM-I-1, PCM-I-2 E PCM-II-2, que apresentam uma relação monômero/cera elevada, apresentam também elevados valores de eficiência da polimerização, próximos a 90\%, pois devido à elevada quantidade de monômero no sistema e a baixa quantidade de cera, o processo de polimerização do MMA possivelmente ocorre via polimerização em emulsão clássica, não ocorrendo o processo de encapsulação da cera. Possivelmente é devido a este fato que se observa o tamanho nanométrico das amostras em questão, pois não ocorrem a formação de cápsulas, ocorre somente a polimerização do monômero.

\section{CONCLUSÕES}

Considerando os resultados de estabilidade e integridade física após secagem em estufa, aspectos morfológicos, tamanho médio de partículas, teor de sólidos, comportamento térmico e potencial de operação em regime cíclico de todas amostras produzidas, conclui-se que o processo de encapsulação segundo o Método II foi mais eficiente para a produção dos encapsulados em escala nanométrica, onde foi possível incorporar grandes quantidades de cera encapsuladas, aumentando assim a entalpia do sistema, sendo eficiente no objetivo incialmente proposto de produção de cápsulas contendo material de mudança de fase capazes de se manter íntegras após processos cíclicos de aquecimento e resfriamento e em escala nanométrica, potencializando assim a capacidade de absorção e liberação de energia térmica devido à ampliação da área de superfície.

\section{AGRADECIMENTOS}

Ao Instituto de Pesquisas Tecnológicas do Estado de São Paulo, pelo apoio e infraestrutura. À Faculdade de Tecnologia de São Paulo.

À Universidade Nove de Julho.

\section{BIBLIOGRAFIA}

[1] PIRASACI, T., GOSWAMI, D.Y., "Influence of design on performance of a latent heat storage system for a direct steam generation power plant”, Applaied Energy, v.162, pp. 644-652, 2016.

[2] YOGEV, R., KRIBUS,A., “Operation strategies and performance of solar termal power plants operating from PCM storage", Solar Energy, pp. 170-180, 2013.

[3] SHARMA, A., TYAGI, V.V., CHEN C.R., et al., "Review on thermal energy storage with phase change materials and applications", Renewable and Sustainable Energy Reviews, Indore, v. 13, pp. 318-345, 2009. 
[4] HWANG, Y.J., LEE, J.K., LEE, C.H., et al., "Stability and thermal conductivity characteristcs of nanofluids", Termochimica Acta, v.455, pp. 70-74, 2007

[5] SÁNCHEZ, P., FERNANDEZ, M. V. S., ROMERO, A., et al., "Development of thermo-regulating textiles using paraffin wax microcapsules”, Thermochimica Acta, Ciudad Real, p.16-21, 2010.

[6] YANG, J., YANG, L., XU, C., et al., "Experimental study on enhancement of thermal energy storage whith phase-change material”, Applied Energy, v.169, pp. 164-176, 2016.

[7] CHANG, C.C., TSAI, Y.L., CHIU, J.J., et al., "Preparation of Phase Change Materials Microcapsules by Using PMMA Network-Silica Hybrid Shell Via Sol-Gel Process”, Wiley InterScience, 2009.

[8] FARID, M.M., KHUDHAIR, A.M., RAZACK, S., et al., "A review on phase change energy storage: materials and applications", Energy Convers Manage, pp.597-615, 2004.

[9] FANG, X.M., ZHANG, Z.G., "A novel montmorillonite-based composite phase change material and its applications in thermal storage building materials”, Energy Buildings, v. 38, p. 377-80, 2006.

[10] ZHANG, Y.P., ZHOU, G.B., LIN, K.P., et al., "Application of latent heat thermal energy storage in buildings: state-of-the-art and outlook”, Building Environ, v. 42, p. 2197, 2007.

[11] AHMET S., CEMIL A., KARAIPEKLI, A., ORHAN, U., "Microencapsulated n-octacosane as phase change material for thermal energy storage", Solar Energy, v. 83, pp. 1757-1763, 2009.

[12] KENISARIN, M., MAHKAMOV, K.., "Solar energy storage using phase change materials", Renewable and Sustainable Energy Reviews, v. 11, pp. 1913-1965, 2007.

[13] KHODADADI, J.M., FAN, L., BABAEI, H., "Thermal conductivity enhancement of nanostructurebased colloidal suspensions utilized as phase change materials for thermal energy storage: a review", Renew. Sustain. Energy Rev., v.24, pp. 418-444, 2013

[14] FAN, L.W., ZHU, Z.Q., XIAO, S.L., et al., "An experimental and numerial investigation of constrained melting heat transfer of a phase change material in a circumferentially finned spherical capsule for thermal energy storage", Applied Thermal Energy, v.100, pp. 1063-1075, 2016.

[15] ZHANG, Y., WEI, J.L., RUI, Y., et al., "Preparation and thermal property of phase change material microcapsules by phase separation”, Material Science Forum, pp. 561-565, 2007.

[16] WANG, T., WANG, S., LUO, R., et al., "Microencapsulation of phase change materials with binary cores and calcium carbonate shell for thermal storage", Applied Materials, v. 171, pp.113-119, 2016.

[17] REGIN, F., SOLANKI, S.C., SAINI, J.S., "An analysis of a packed bed latent heat thermal energy storage system using PCM capsules: numerical investigation”, Applied Energy, v.34, pp. 1765-1773, 2009.

[18] ZHAO,W., NETI, S., OZTEKIN, A., "Heat transfer analysis of encapsulated phase change materials", Applied Thermal Engineering, v. 50, pp. 143-151, 2013. 